\title{
Review Article \\ Natural Bioactive Compounds: Alternative Approach to the Treatment of Glioblastoma Multiforme
}

\author{
Vilas Desai and Alok Bhushan \\ Department of Pharmaceutical Sciences, Jefferson College of Pharmacy, Thomas Jefferson University, Philadelphia, PA 19107, USA
}

Correspondence should be addressed to Alok Bhushan; alok.bhushan@jefferson.edu

Received 6 June 2017; Accepted 17 October 2017; Published 20 November 2017

Academic Editor: Marta M. Alonso

Copyright (C) 2017 Vilas Desai and Alok Bhushan. This is an open access article distributed under the Creative Commons Attribution License, which permits unrestricted use, distribution, and reproduction in any medium, provided the original work is properly cited.

Glioblastoma multiforme (GBM) is the most frequent, primary malignant brain tumor prevalent in humans. GBM characteristically exhibits aggressive cell proliferation and rapid invasion of normal brain tissue resulting in poor patient prognosis. The current standard of care of surgical resection followed by radiotherapy and chemotherapy with temozolomide is not very effective. The inefficacy of the chemotherapeutic agents may be attributed to the challenges in drug delivery to the tumor. Several epidemiological studies have demonstrated the chemopreventive role of natural, dietary compounds in the development and progression of cancer. Many of these studies have reported the potential of using natural compounds in combination with chemotherapy and radiotherapy as a novel approach for the effective treatment of cancer. In this paper, we review the role of several natural compounds individually and in combination with chemotherapeutic agents in the treatment of GBM. We also assess the potential of drug delivery approaches such as the Gliadel wafers and role of nanomaterial based drug delivery systems for the effective treatment of GBM.

\section{Introduction}

Human gliomas constitute the major form of primary brain tumors $[1,2]$. These tumors can be classified as low-grade gliomas, glioblastomas, or anaplastic astrocytomas, based on the degree of invasiveness and pathology of the tumor $[2,3]$. The most malignant form of astrocytoma is called glioblastoma multiforme (GBM), typically characterized by an increased angiogenesis, invasion of normal brain tissues, and necrosis, and has the worst prognosis [4-6]. At a cellular level, GBM is poorly differentiated, with round or pleomorphic cells that are multinucleated and anaplastic [6-8]. Depending on origin, it is categorized as either primary GBM, which arises de novo from the glial cells, or secondary GBM, which arises from preexisting lower grade astrocytoma [6,9]. The hallmark feature of primary GBM is overexpression of the EGFR gene and loss of heterozygosity of PTEN while secondary GBM exhibits loss of $\mathrm{p} 53$ and overexpression of PGDF [8-10]. Although the causes of GBM are relatively unclear, its risk may be increased by certain factors (i.e., family history, brain trauma, and immune system alteration) and conditions (i.e., Li-Fraumeni's syndrome) [5,6]. The current therapy includes surgical resection of the tumor, radiotherapy, and adjuvant chemotherapy with temozolomide $[3,9,11]$. The complete surgical resection of gliomas is difficult, and due to local invasion and infiltration of normal tissue the tumor recurs leading to death of patients with glioblastoma $[3,9]$. The efficacy of chemotherapy is further decreased by the presence of the blood brain barrier that limits the delivery of chemotherapeutics to the brain $[9,11]$. The current therapy minimally improves the median survival time of patients from 12 months to $\sim 14.6$ months. The 5 -year survival rate of treated patients is $<10 \%[1,3,9]$. Hence, there is an imperative need for the development of novel, targeted, and effective therapies for GBM.

\section{Natural Compounds in Cancer}

Conventional cancer therapies such as chemotherapy and radiotherapy essentially exert their cytotoxic effects by damaging the DNA of cancer cells $[12,13]$. However, limitations exist with these treatments when used as single modalities due to the high heterogeneity in solid tumors and the deregulation of several cell signaling cascades [14-18]. GBM is particularly difficult to treat because of the heterogeneity of the tumor, its highly aggressive infiltration into the surrounding tissues, and the presence of blood brain barrier [3, 4]. Multimodality treatment approaches can be an effective 
strategy for GBM wherein different therapies or therapeutic agents with distinct molecular mechanisms are combined to exert an improved cytotoxic effect on cancer cells [10, 11]. In particular, multimodal therapy can work effectively by sensitizing cancer cell DNA by one agent to the damaging effects of the other $[12,13,19]$.

In recent years, several epidemiological studies have investigated the role of natural, dietary compounds in influencing the development, progression, and metastasis of cancer [19-22]. A wide range of natural compounds have been recognized for their antioxidant nature and for their cancer chemopreventive potential, including soy isoflavones, curcumin, epigallocatechin, resveratrol, and retinoids [2325]. The main objective of this review paper is to discuss the role of these natural compounds in enhancing the efficacy of chemotherapeutic drugs in GBM treatment as reported by a number of studies. We also review the significance of novel drug delivery methods such as the Gliadel wafers and the emerging role of nanomedicine in drug delivery for GBM treatment.

2.1. Isoflavones in Glioma. Several studies have demonstrated the benefits of consumption of a plant-based diet of fruits and vegetables [13-19]. Soy isoflavones, such as genistein, daidzein, and biochanin $\mathrm{A}$, are natural polyphenolic compounds with potent antioxidant, anti-inflammatory, and weak estrogenic properties [18, 19]. Isoflavones are usually derived from soy and soy-based products but are also found in chick peas, nuts, grain products, and red clover $[12,15]$. They have been implicated in cancer prevention, based on the epidemiological reports that South Asian populations have heavy consumption of soy and soycontaining foods and low incidence of cancer and cardiovascular diseases compared to the people in Western civilizations $[14,19]$. Furthermore, they also reportedly have beneficial effects in endocrine-responsive cancer, osteoporosis, menopause, and coronary heart diseases [21, 22]. Interest in soy isoflavones has been renewed by the research showing the estrogen-like ring in their structure, indicating that they may be a better alternative to synthetic selective estrogen receptor modulators (SERMs) currently used in breast cancer prevention and hormone replacement therapy [20, 21, 23].

The intestinal microflora in the body converts isoflavones to isoflavonoids which reportedly play a critical, preventive role in mutation and promotional phases of cancer progression $[21,22]$. Cancer chemopreventive properties of isoflavones in improving the efficacy of chemotherapy and radiotherapy have been demonstrated in vitro and in vivo, and a number of clinical trials are focusing on the antiinvasive and antiangiogenic properties of these compounds for treatment of GBM [18, 21, 23].

2.1.1. Genistein. Genistein is one type of soy isoflavone that has shown promise as a chemopreventive agent due to its antioxidant and anti-inflammatory properties and its ability to potently inhibit angiogenesis and metastasis [16, $18,20]$. Our previous studies have reported its ability to inhibit invasion in a coculture model of GBM by inhibiting tyrosine kinase EGFR [24]. Other studies have shown that genistein prevents the hypermethylation of promoter regions of tumor suppressor genes (i.e., p21, BRCA1) by inhibiting DNA methyltransferases in different cancer types $[20,22]$. In other research, genistein inhibited the expression of apurinic/apyrimidinic (AP) endonuclease 1 (APE1) in prostate cancer cells in a dose dependent manner [18, 23]. APE1 is an enzyme involved in the DNA base excision repair (BER) and redox signaling, and elevated levels have been correlated to resistance to chemotherapy $[13,15,16]$. Genistein is also a known inhibitor of protein tyrosine kinase (PTK), and it competes with ATP to bind to the tyrosine kinase domain thereby inhibiting the activation of tyrosine kinase-mediated downstream signaling processes $[20,22$, 24]. A recent study on genistein showed its inhibitory effects on telomerase activity and subsequent cell cycle arrest in radiosensitive brain tumor cells $[15,17,22]$. There have been several clinical studies at phases I, II, and III on genistein as an adjuvant compound on patients undergoing chemotherapy or radiotherapy for prostate, bladder, or breast cancer [25]. In a study on 20 prostate cancer patients treated with pure genistein, it was observed that there were no genotoxic effects or any change in micronuclei number and no damage to the chromosomes compared to the nontreated lot [26]. A wide range of clinical studies on isoflavones have demonstrated a good safety profile even at maximal dose which is encouraging for the population [26]. However, in clinical studies, various factors such as the stage or type of cancer and differences in the metabolic state of individuals play a critical role in determining the outcome of such studies. Nonetheless, the results of a number of studies on isoflavones in cancer are encouraging and hold a potential for further research.

2.1.2. Biochanin A. Biochanin A, a bioactive isoflavone and a prodrug or methoxy form of genistein that is found in red clover, has been shown to inhibit the incidence and growth of LNCaP xenograft tumors in athymic mice [21, 23]. Earlier studies showed that it has chemopreventive and anticancer potential against glioblastoma, breast cancer, prostate cancer, and oral cancer cells $[11,17,23,24]$. Biochanin A is documented to be less mutagenic than genistein, and hence it may be a more suitable candidate for use as a chemopreventive agent $[14,18]$. It is rapidly degraded to genistein, genistein conjugates, and biochanin A conjugates, which confer anticancer properties to the compound [20,23]. It has tyrosine kinase inhibitory properties that are weaker than those of genistein and inhibits the growth of breast, colon, and prostate cancer cells in vitro $[11,17,18,27]$. Studies have also shown that biochanin A has inhibitory potential on the development of lung tumors induced in mice by benzo(a)pyrene [23].

Both biochanin $\mathrm{A}$ and genistein have been found to inhibit both serum and EGF-stimulated growth of human prostate cancer cells $[17,18]$. Our earlier studies showed that genistein and biochanin A inhibit the invasion of glioblastoma cells by inhibiting matrix metalloproteases (MMPs) [11]. Both isoflavones have also been found to enhance 
the efficacy of rapamycin in inhibiting the mTOR pathway in glioblastoma cells and their rapamycin-induced feedback upregulation of AKT [24].

2.2. Resveratrol. Resveratrol (trans-3, $4^{\prime}, 5$-trihydroxystilbene) (RSV) is a naturally occurring polyphenolic phytoalexin present in grapes, mulberries, peanuts, and vegetables [27, 28]. It has attracted substantial attention in recent years due to its antioxidant and anti-inflammatory properties, which give it efficacy in treating cardiovascular diseases, and due to its neuroprotective effects in penetrating the blood brain barrier, which give it efficacy for treating ischemia and hypoxia [29]. The chemopreventive and antioncogenic effects of RSV have been reported in several cancer types [27-29]. Researchers found that RSV prevented the development of skin cancer in mice during the different stages of carcinogenesis [27] and inhibited DMBA-induced mammary carcinogenesis [28]. Clinical trial studies in patients with colorectal cancer have shown that RSV hinders tumor cell proliferation and increases caspase- 3 in the malignant hepatic tissue compared to the placebo patients $[30,31]$. In a phase I trial study in healthy volunteers, it was observed that ingesting RSV ( 0.5 to $5 \mathrm{~g}$ /day for 29 days) led to a decrease in systemic IGF-1 and IGFBP-3 levels, which may contribute to the antiproliferative activity of RSV [32].

In earlier studies by Gangemi et al., RSV was found to induce apoptosis by activating caspase- 3 in a human glioma cell line U251 [33]. Further, Ryu et al. demonstrated that RSV reduced the invasion in U373MG glioma cells induced via TNF- $\alpha$, by regulating the NF- $\kappa$ B activation and expression of upa/upar [34]. A study by Jang et al. reported the chemopreventive and anticarcinogenic effects of RSV in the different stages of carcinogenesis, namely, initiation, promotion, and progression [28]. RSV causes cell death in GBM cells through mechanisms such as autophagy, apoptosis, and senescence $[28,35]$. It exerts its cytotoxic and cytostatic effects in cancer cells by modulating the cell cycle at specific points including an $S$ phase arrest in medulloblastoma cells [35].

A study by Filippi-Chiela et al. on several GBM cell lines found that RSV potentiates the toxicity of TMZ in combination treatment mainly by inhibiting TMZ-induced G2/M arrest followed by induction of senescence and MC [35]. This G2/M arrest was p53 independent, as it was observed in all glioma cells tested, including p 53 mutant cells U251 and U138 [35]. Cilibrasi et al. investigated the effects of RSV on seven glioma stem cell (GSC) lines derived from GBM patients. They observed that RSV inhibited the cell proliferation, increased cell mortality, and reduced motility of the cells by modulating the Wnt signaling pathway [3638]. These findings clearly suggest that RSV possesses potent therapeutic properties and may work as effective adjuvant molecules in combination therapy of cancer.

2.3. Epigallocatechin Gallate. Epigallocatechin gallate (EGCG) is a major polyphenolic green tea component and a major catechin in green tea $[39,40]$. EGCG has been extensively investigated for its potential chemopreventive and chemosensitizing properties in a variety of malignant cancers $[41,42]$. It has the ability to bind to GRP78, a key prosurvival component of ER stress response system, and inactivate its antiapoptotic function, an interaction that makes cancer cells more chemosensitive [41]. A number of studies have shown that EGCG increases the sensitivity of different cancer types to different apoptotic drugs, such as 5-fluorouracil, gemcitabine, or taxol, in vitro [42-44] and to doxorubicin and paclitaxel in vivo $[44,45]$. Studies have also reported that EGCG can reverse drug resistance by inducing apoptosis and inhibiting P-gp expression and ABCG2 in drug-resistant cancer cells of the ovaries, breast, and lung $[40,42]$. There have been reports of the inhibition of Wnt signaling by EGCG in breast cancer cells and an upregulation of p53 transcriptional activity in LNCaP cells [42, 46, 47]. In a double-blind placebo-controlled clinical trials with green tea catechins (GTCs) against prostate cancer, it was shown that GTCs were safe and effective in treating premalignant lesions before the prostate cancer developed [48]. In another phase II study among 42 patients of androgen independent prostate carcinoma, a minimal antineoplastic activity with a decline in prostate specific antigen (PSA) levels was reported [49]. However, there have been a few positive reports with EGCG inducing apoptosis in leukemic B-cells in majority of patients of chronic lymphocytic leukemia (CLL) and patients with low-grade B-cell malignancies [50].

A recent study by Zhang et al. reported that EGCG reduced U87 and C6 GSLC (Glioma Stem-Like Cells) viability, neurosphere formation capability, and migration. EGCG also sensitized GSLCs to temozolomide, a phenomenon associated with downregulation of P-gp in vitro $[40,43]$. EGCG was observed to induce apoptosis in U87 GSLCs by reducing Akt phosphorylation, inactivating antiapoptotic protein Bcl-2, upregulating the apoptosis-promoting protein Bax, and cleaving PARP $[51,52]$. Since gliomas are sensitive to apoptosis through the downregulation of Bcl-2 family $[47,51]$, EGCG is potentially an effective molecule to target GSLCs associated with inhibition of an Akt-related pathway [52]. Although it remains unclear whether EGCG can achieve chemosensitization of cancer cells across the blood brain barrier, which would be required for the treatment of malignant gliomas, these studies provided clear evidence that polyphenol compounds like EGCG can significantly augment the chemotherapeutic efficacy of cancer drugs in a combination treatment both in vitro and in vivo.

2.4. Retinoids. Retinoids have also been found to potentially enhance the efficacy of chemotherapy and radiotherapy in GBM. Retinoids are a class of chemical compounds that are related to vitamin A and are fat soluble [53, 54]. Retinol is transported from the liver to the target tissues as retinolbinding protein and is then enzymatically converted to retinaldehyde and consequently to retinoic acid $[53,55]$. The retinoid signaling pathways play an important role in neurogenesis, dendritic growth of hippocampal neurons, and higher cognitive functions $[53,54]$. Studies have shown that retinoids strongly inhibit the cell proliferation and migration in primary cultures of human glioblastoma multiforme [54, 55]. Retinoids can successfully induce differentiation but cannot induce apoptosis. However a synthetic analog of alltrans retinoic acid (ATRA), N-(4-hydroxyphenyl) retinamide 
(4-HPR), exhibits both antiproliferative and proapoptotic effects [55]. 4-HPR is a much more effective and relatively less toxic compound than ATRA, and it is proven to be effective even in ATRA-resistant cells $[55,56]$.

In one notable study in which 4-HPR treatment was used in combination with the knockdown of survivin, a prosurvival protein overexpressed in GBM that has been positively correlated with GBM cell proliferation, around $80 \%$ of the cells were apoptotic and in vivo angiogenesis studies showed a noticeable decrease in tumor vascularization [54, 56, 57]. In a similar study, when GBM cells were treated with retinoids, differentiation of astrocytes was induced and telomerase activity was inhibited, allowing for an increased sensitivity to interferon- $\gamma$ therapy [58]. Moreover, treatments with retinoids have been shown to reduce levels of inflammatory factors, potentially making the GBM cells sensitive to radiotherapy $[53,54]$.

\subsection{Other Notable Natural Compounds with}

\section{Promising Anticancer Activity against GBM}

2.5.1. Cannabis and Cannabinoids. Cannabis sativa L. and its derivative compounds cannabinoids have been reported to have a broad range of pharmacological effects mediated specifically by two plasma membrane receptors (CB1 and CB2) [59-62]. $\Delta^{9}$-Tetrahydrocannabinol (THC) is the most potent and abundant endocannabinoid in cannabis that can bind to and activate specific cell receptors $[60,63]$. Several studies have reported the efficacy of cannabinoids in the treatment of pain, inflammation, depression, neurological disorders, and cancer [59, 60, 62-66]. The anticancer potential of cannabinoids against gliomas may be mediated through the CB1 receptor which is densely expressed in the brain as a seven-transmembrane domain $G$ proteincoupled receptor and is activated by the receptor agonist $\Delta^{9}$ THC in vitro and in vivo in the treatment of glioblastoma (GBM) [61-66]. The activation of $\mathrm{CB1}$ and $\mathrm{CB} 2$ by $\Delta^{9}-$ THC impairs cancer cell proliferation and invasion, induces apoptosis by ceramide accumulation in culture, and also reduces the tumor volume in animals $[59,63] . \Delta^{9}$-THC in combination with temozolomide (TMZ) has shown a robust anticancer activity in TMZ-sensitive as well as TMZ-resistant tumors in glioma xenografts [65]. A phase I clinical study in brain tumor patients has reported the safety of direct intratumoral injection of tetrahydrocannabinol in recurring GBM [66]. However, to validate the efficacy of cannabis in preventing gliomas and the methods of administering the other derivatives of cannabis safely, more clinical trials are needed [66]. In recognition of the promising anticancer potential of $\Delta^{9}$-THC in the preclinical studies with a fair safety profile, it has become an important therapeutic target for the treatment of GBM and has also prompted a human clinical trial $[61,66]$.

2.5.2. Neurostatin. Neurostatin is a natural glycosphingolipid, an O-acetylated ganglioside GD1b, present in the mammalian brain, and shows strong inhibition of astroblast and astrocytoma division [66, 67]. Although it exhibits a high inhibitory activity against gliomas, it is relatively less abundant in the brain $[68,69]$. However, neurostatin has now been extensively purified from the ganglioside extracts of rats and bovine and porcine brain and is observed to be cytostatic against C6 glioma cells and grade III and IV human astrocytoma cells $[66,69]$. In particular neurostatin has been shown to impair glioma cell proliferation in vivo by inducing cell cycle arrest and potentiating the immune cell response to the tumor through the activation of CD4+ and CD8+ lymphocytes [67]. Valle-Argos et al. reported that the anticancer activity of neurostatin in vitro and in vivo in gliomas is mediated through the arrest of cell cycle progression, while interfering with the angiogenic and invasive mechanisms $[66,67]$. Neurostatin was shown to inhibit the expression of cell cycle promoters (cyclins) and CDKs while upregulating cell cycle inhibitors such as p21 and p27 $[67,70]$. The promitogenic pathways of MAPK and PI3K were also reportedly blocked through the inhibition of EGFR signaling [67, 70]. Gangliosides like neurostatin are ubiquitous molecules with potent and specific biological actions, and the preclinical studies strongly indicate that it is a promising therapeutic candidate in the treatment of gliomas $[69,70]$.

2.5.3. Bipolaris setariae Fungi. Bipolaris is a genus of dematiaceous hyphomycetes with more than 100 species [71]. Ophiobolin A (OP-A), a sesterterpenoid that is produced by the plant pathogenic fungi, was purified from the culture extract of Drechslera gigantea and characterized to be an effective phytotoxin [72, 73]. Further studies on the compound showed a broad spectrum of biological and pharmacological characteristics including anticancer activity $[72,73]$. A number of studies have reported the anticancer activity of OP-A with an $\mathrm{IC}_{50}$ in micromolar concentration range against different glioblastoma and neuroblastoma cells $[73,74]$. A study by Bury et al. reported that OP-A caused marked changes in the organization of the actin cytoskeleton and induced paraptosis through the disruption of internal potassium ion homeostasis in glioblastoma cells [73]. The reported studies indicate that the OP-A causes mitochondrial dysfunction and ER stress, impairs cell cycle progression, and inhibits multiple oncogenic signaling pathways in glioblastoma cells $[73,74]$. Table 1 shows some of the natural compounds used in the treatment of GBM and their reported mechanism of action in in vitro and in vivo studies. OP-A thus represents a class of natural compounds that can be used to combat cancer types exhibiting different levels of resistance to proapoptotic stimuli $[72,74]$.

\section{New Approaches to Drug Delivery}

3.1. Gliadel Wafers. Despite surgical resection of GBM tumor followed by radiotherapy and chemotherapy (i.e., carmustine (BCNU) and temozolomide), recurrences are inevitable [75, 76]. Effective delivery of drugs to the tumor is a major challenge due to systemic toxicities and the need for transport across the blood brain barrier [75-77]. Gliadel wafers offer a novel system for delivering chemotherapeutic agents to GBM cells. Approved for treating recurrent glioblastoma by FDA in 1995, Gliadel wafers are biodegradable polymers loaded 
TABLE 1: Natural compounds and their mechanism of action in GBM and other cancer cells.

\begin{tabular}{ll}
\hline $\begin{array}{l}\text { Natural } \\
\text { compounds }\end{array}$ & Target mechanisms \\
$\begin{array}{l}\text { Isoflavones } \\
\text { (biochanin A, } \\
\text { genistein, etc.) }\end{array}$ & $\begin{array}{l}\text { Inhibits MMPs and thus invasion \& metastasis. } \\
\text { Inhibits tyrosine kinase mediated downstream signaling. }\end{array}$ \\
\hline Resveratrol & $\begin{array}{l}\text { Induces apoptosis by activating caspase-3. } \\
\text { Modulates cell cycle at specific points. }\end{array}$ \\
\hline $\begin{array}{l}\text { Epigallocatechin } \\
\text { A (EGCG) }\end{array}$ & $\begin{array}{l}\text { Binds to GRP78 (prosurvival component of ER stress response system) and inactivates its antiapoptotic } \\
\text { function. Inhibits P-gp expression thus reversing drug resistance in cancer cells. }\end{array}$ \\
\hline Retinoids & $\begin{array}{l}\text { Reduces levels of inflammatory factors making cancer cells sensitive to radiotherapy. Increases sensitivity to } \\
\text { specific therapies such as interferon- } \gamma \text { therapy by inhibiting telomerase activity. }\end{array}$ \\
\hline Cannabis & $\begin{array}{l}\text { Induces apoptosis by sustained accumulation of ceramide that also upregulates the ERK activity. } \\
\text { Causes ER stress and inhibition of pAkt/mTOR leading to autophagy-mediated cell death. }\end{array}$ \\
\hline Neurostatin & $\begin{array}{l}\text { Inhibits cell cycle progression (suppresses cyclins and CDKs and promotes inhibitors such as p21 \& p27). } \\
\text { Impairs promitogenic pathways of MAPK and PI3K by blocking EGFR signaling. }\end{array}$ \\
\hline $\begin{array}{l}\text { Bipolaris } \\
\text { setariae fungi }\end{array}$ & $\begin{array}{l}\text { Disrupts the potassium ion homeostasis causing mitochondrial dysfunction and ER stress. Impairs cell cycle } \\
\text { progression. }\end{array}$ \\
\hline
\end{tabular}

with carmustine $[77,78]$. They are implanted at the resection site, and the drug is gradually released over a period of 2-3 weeks as the wafer polymer degrades [78-80]. In a placebocontrolled, double-blind phase 3 trial in 2003, 2-year survival was higher among glioblastoma patients treated with Gliadel wafers $(15.8 \%)$ than patients who received placebo $(8.3 \%)$; median survival was 13.8 months in the patients treated with the Gliadel wafer and 11.6 months in the placebo group [7981].

Another study treated glioma patients who had undergone surgery with Gliadel wafers soaked with temozolomide $[80,81]$. The patients treated with Gliadel wafer and temozolomide survived 20.7 months (median) and the 2year survival rate was $36 \%$ [80]. Several other studies have reported that when Gliadel wafers are implanted in the resection cavity of malignant gliomas, local drug delivery is improved and systemic side effects are reduced in recurrent glioma treatment. However, there have also been reports that use of Gliadel wafers resulted in adverse effects, including cerebral edema, surgical site infection, perioperative seizures, and severe hydrocephalus leading to death [77-79, 82]. Hence, the practice of using Gliadel wafers for the treatment of GBM needs to be reevaluated.

\subsection{Nanomedicine and Drug Delivery across the Blood Brain} Barrier. During the last couple of decades, nanomedicine has progressed significantly, especially in the field of cancer therapeutics. A key feature of the nanomaterials is their size which operate at the same scale as the biological molecules and pathways. This means that nanoscale materials can be designed to interact with biological entities in a direct, efficient, and precise manner and that they can help us understand biological processes and pathways at the molecular level $[83,84]$. Nanometerials can be designed to circumvent or cross the $\mathrm{BBB}$ and hence may serve as an effective drug delivery system in GBM treatment.

Treatment for GBM is often impaired because therapeutic agents cannot be delivered adequately to the target tumor cells; the problem is further compromised by the presence of blood brain barrier (BBB) $[85,86]$. Blood brain barrier $(\mathrm{BBB})$ is a unique anatomical structure mainly formed by tight junctions and adherence junctions between the brain endothelial cells that selectively regulate the flow of ions, nutrients, and cells into the brain [85, 87]. This restricted permeation of drugs to the GBM cells, allowing a fraction of cells called cancer stem cells (CSCs) to evade drug cytotoxicity and develop therapeutic resistance [86, 88, 89]. Further, the poor delivery of chemotherapeutic agents can also be attributed to their large size, hydrophobic nature, and their efflux by the MDR efflux pumps expressed by BBB and tumor cells $[87,88]$. Thus, targeted, effective, therapeutic regimens are needed that can cross the $\mathrm{BBB}$ and reach their target in the brain $[90,91]$. A few proposed strategies include passive permeation of lipidated drugs, development of prodrugs that can hijack the transport mechanism of $\mathrm{BBB}$, and drug-loaded nanocarriers [87, 88, 92, 93].

Apart from a limited number of liposoluble, small molecules, most of the other molecules need a specific transport system to cross the $\mathrm{BBB}[84,92]$. Hence, a more selective and targeted approach such as the nanoparticles based system can be employed to design targeted therapies. Colloidal systems such as the nanoparticle system allow for the design of nanocarriers with surface properties to overcome the biochemical/biophysical barriers, and can be tailored to deliver the drugs across the BBB. Moreover, the surface properties of the nanocarriers can be modified for a selective, controlled drug release with minimal side effects and increased efficacy $[84,94,95]$. Nanomaterial based chemotherapeutic agents such as the liposomes, dendrimers, or polymeric micelles are reported to circumvent the BBB and reach the target site of action [87, 88, 96, 97]. Several nanomaterials-based drugs have been evaluated for the treatment of GBM and other cancers. Table 2 features some of these drugs which have been approved by the regulatory authority and are in clinical use for GBM as well as other types of cancers [85-100]. 


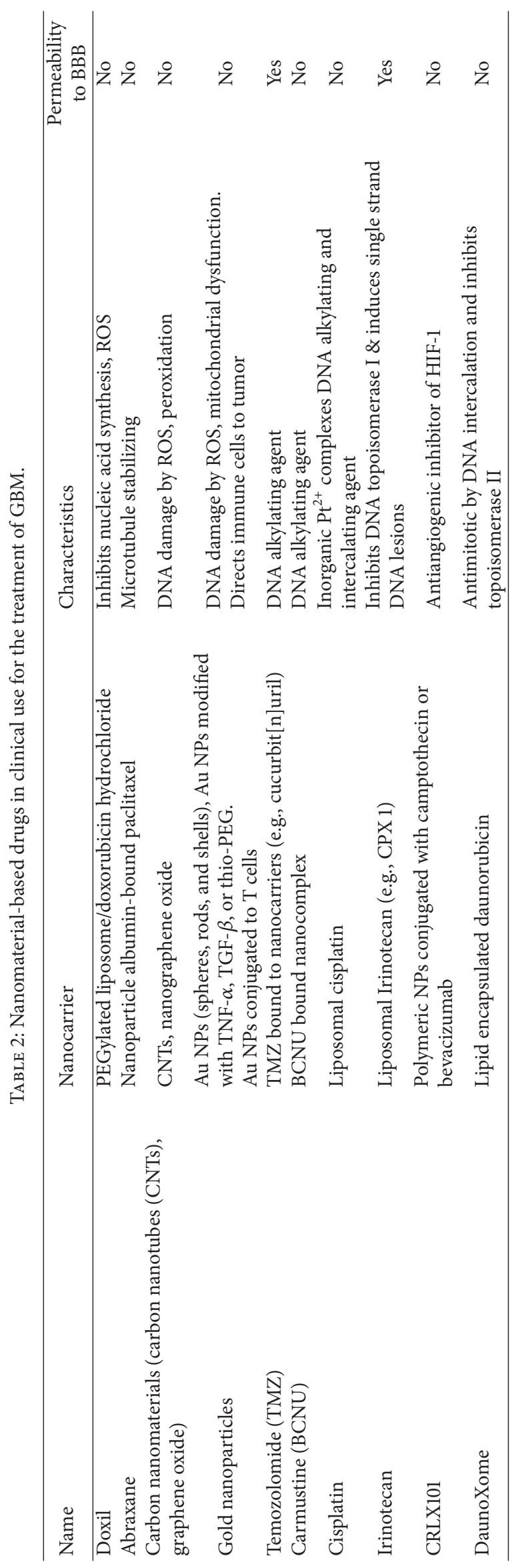


Glioblastomas exhibit a high degree of heterogeneity and the integrity of $\mathrm{BBB}$ varies, with high-grade gliomas showing a leaky BBB and low-grade gliomas showing an intact BBB [98-100]. Hence, the GBM therapies need to be designed to identify disease-related changes in the $\mathrm{BBB}$ and to tailor the drug or drug nanocarriers accordingly $[99,100]$. Ideally, the carrier agents should be cationic for optimal vascular absorption, small, stable in biological fluids, and tailored to carry a large drug payload $[97,99,100]$. The physiological and pathophysiological status of the gliomas also needs to be considered for optimization of any chemotherapeutic agent for GBM.

\section{Conclusions and Future Directions}

The current standard therapy of GBM has shown little promise and there have been efforts to design novel, targeted, effective therapeutics. Moreover, the current chemotherapy may also be a cause of drug resistance in GBM treatment as it severely destabilizes the cell metabolism and cell signaling network. Here we have reviewed a number of studies that report the role of various natural, dietary compounds that show significant improvement in the efficacy of chemotherapeutics in combination therapy of GBM both in vitro and in vivo. Glioblastoma is a highly heterogeneous and often fatal cancer, and attacking its chemoresistance is a critical step in combating its growth. Several of the studies reviewed emphasize the relative nontoxicity of the natural compounds and improvement in efficacy of combination therapy at a lower dosage level. We have also reviewed the role of Gliadel wafers after surgery in the local delivery of drugs such as BCNU and temozolomide in brain tumor treatments. The main challenge of GBM treatment is the inadequacy of drug delivery due to the presence of $\mathrm{BBB}$. We have discussed the role of nanocarriers and nanomedicine in overcoming the obstacle of $\mathrm{BBB}$ to improve the efficacy of drug delivery and thus the GBM treatment. The studies reviewed above clearly demonstrate the anticancer potential of these natural compounds and indicate that they can be an alternative approach to a more effective and relatively nontoxic GBM treatment.

\section{Conflicts of Interest}

The authors declare that there are no conflicts of interest regarding the publication of this paper.

\section{References}

[1] J. L. Villano, T. E. Seery, and L. R. Bressler, "Temozolomide in malignant gliomas: current use and future targets," Cancer Chemotherapy and Pharmacology, vol. 64, no. 4, pp. 647-655, 2009.

[2] A. V. Knizhnik, W. P. Roos, T. Nikolova et al., "Survival and death strategies in glioma cells: autophagy, senescence and apoptosis triggered by a single type of temozolomide-induced DNA damage," PLoS ONE, vol. 8, no. 1, Article ID e55665, pp. $1-12,2013$.
[3] J. Zhang, M. F. G. Stevens, and T. D. Bradshaw, "Temozolomide: mechanisms of action, repair and resistance," Current Molecular Pharmacology, vol. 5, no. 1, pp. 102-114, 2012.

[4] C. J. Sandberg, G. Altschuler, J. Jeong et al., "Comparison of glioma stem cells to neural stem cells from the adult human brain identifies dysregulated Wnt- signaling and a fingerprint associated with clinical outcome," Experimental Cell Research, vol. 319, no. 14, pp. 2230-2243, 2013.

[5] S. K. Singh, C. Hawkins, I. D. Clarke et al., "Identification of human brain tumour initiating cells," Nature, vol. 432, no. 7015, pp. 396-401, 2004.

[6] T. Aoki, N. Hashimoto, and M. Matsutani, "Management of glioblastoma," Expert Opinion on Pharmacotherapy, vol. 8, no. 18, pp. 3133-3146, 2007.

[7] J. M. Gallego, J. A. Barcia, and C. Barcia-Mariño, "Fatal outcome related to carmustine implants in glioblastoma multiforme," Acta Neurochirurgica, vol. 149, no. 3, pp. 261-265, 2007.

[8] K. K. Jain, "Use of nanoparticles for drug delivery in glioblastoma multiforme," Expert Review of Neurotherapeutics, vol. 7, no. 4, pp. 363-372, 2007.

[9] M. C. Chamberlain, "Temozolomide: therapeutic limitations in the treatment of adult high-grade gliomas," Expert Review of Neurotherapeutics, vol. 10, no. 10, pp. 1537-1544, 2010.

[10] S. Vinogradov and X. Wei, "Cancer stem cells and drug resistance: the potential of nanomedicine," Nanomedicine, vol. 7, no. 4, pp. 597-615, 2012.

[11] S. Puli, J. C. K. Lai, and A. Bhushan, "Inhibition of matrix degrading enzymes and invasion in human glioblastoma (U87MG) cells by isoflavones," Journal of Neuro-Oncology, vol. 79, no. 2, pp. 135-142, 2006.

[12] A. R. M. R. Amin, O. Kucuk, F. R. Khuri, and D. M. Shin, "Perspectives for cancer prevention with natural compounds," Journal of Clinical Oncology, vol. 27, no. 16, pp. 2712-2725, 2009.

[13] N. P. Gullett, A. R. M. Ruhul Amin, S. Bayraktar et al., "Cancer prevention with natural compounds," Seminars in Oncology, vol. 37, no. 3, pp. 258-281, 2010.

[14] H. Adlercreutz and W. Mazur, "Phyto-oestrogens and Western diseases," Annals of Medicine, vol. 29, no. 2, pp. 95-120, 1997.

[15] G. G. Hillman and V. Singh-Gupta, "Soy isoflavones sensitize cancer cells to radiotherapy," Free Radical Biology \& Medicine, vol. 51, no. 2, pp. 289-298, 2011.

[16] F. H. Sarkar and Y. Li, "Using chemopreventive agents to enhance the efficacy of cancer therapy," Cancer Research, vol. 66, no. 7, pp. 3347-3350, 2006.

[17] I. U. Ahmad, J. D. Forman, F. H. Sarkar et al., "Soy isoflavones in conjunction with radiation therapy in patients with prostate cancer," Nutrition and Cancer, vol. 62, no. 7, pp. 996-1000, 2010.

[18] J. J. Raffoul, F. H. Sarkar, and G. G. Hillman, "Radiosensitization of prostate cancer by soy isoflavones," Current Cancer Drug Targets, vol. 7, no. 8, pp. 759-765, 2007.

[19] A. Bapat, M. L. Fishel, and M. R. Kelley, "Going ape as an approach to cancer therapeutics," Antioxidants \& Redox Signaling, vol. 11, no. 3, pp. 651-667, 2009.

[20] F. H. Sarkar, Y. Li, Z. Wang, and D. Kong, "Cellular signaling perturbation by natural products," Cellular Signalling, vol. 21, no. 11, pp. 1541-1547, 2009.

[21] M. Goodman, R. M. Bostick, O. Kucuk, and D. P. Jones, “Clinical trials of antioxidants as cancer prevention agents: past, present, and future," Free Radical Biology \& Medicine, vol. 51, no. 5, pp. 1068-1084, 2011. 
[22] S. Banerjee, Y. Li, Z. Wang, and F. H. Sarkar, "Multi-targeted therapy of cancer by genistein," Cancer Letters, vol. 269, no. 2, pp. 226-242, 2008.

[23] V. Singh-Gupta, M. C. Joiner, L. Runyan et al., "Soy isoflavones augment radiation effect by inhibiting APE1/ref-1 DNA repair activity in non-small cell lung cancer," Journal of Thoracic Oncology, vol. 6, no. 4, pp. 688-698, 2011.

[24] S. Puli, A. Jain, J. C. K. Lai, and A. Bhushan, "Effect of combination treatment of rapamycin and isoflavones on mtor pathway in human glioblastoma (U87) cells," Neurochemical Research, vol. 35, no. 7, pp. 986-993, 2010.

[25] M. K. Virk-Baker, T. R. Nagy, and S. Barnes, "Role of phytoestrogens in cancer therapy," Planta Medica, vol. 76, no. 11, pp. 1132-1142, 2010.

[26] W. Miltyk, C. N. Craciunescu, L. Fischer et al., "Lack of significant genotoxicity of purified soy isoflavones (genistein, daidzein, and glycitein) in 20 patients with prostate cancer.," American Journal of Clinical Nutrition, vol. 77, no. 4, pp. 875882, 2003.

[27] V. Sehdev, C. K. J. Lai, and A. Bhushan, "Biochanin A Modulates Cell Viability, Invasion, and Growth Promoting Signaling Pathways in HER-2-Positive Breast Cancer Cells," Journal of Oncology, vol. 2009, pp. 1-10, 2009.

[28] M. Jang, L. Cai, G. O. Udeani et al., "Cancer chemopreventive activity of resveratrol, a natural product derived from grapes," Science, vol. 275, no. 5297, pp. 218-220, 1997.

[29] C. Gill, S. E. Walsh, C. Morrissey, J. M. Fitzpatrick, and R. W. G. Watson, "Resveratrol sensitizes androgen independent prostate cancer cells to death-receptor mediated apoptosis through multiple mechanisms," The Prostate, vol. 67, no. 15, pp. 16411653, 2007.

[30] K. R. Patel, V. A. Brown, D. J. L. Jones et al., "Clinical pharmacology of resveratrol and its metabolites in colorectal cancer patients," Cancer Research, vol. 70, no. 19, pp. 7392-7399, 2010.

[31] L. M. Howells, D. P. Berry, P. J. Elliott et al., "Phase I randomized, double-blind pilot study of micronized resveratrol (SRT501) in patients with hepatic metastases-safety, pharmacokinetics, and pharmacodynamics," Cancer Prevention Research, vol. 4, no. 9, pp. 1419-1425, 2011.

[32] V. A. Brown, K. R. Patel, M. Viskaduraki et al., "Repeat dose study of the cancer chemopreventive agent resveratrol in healthy volunteers: safety, pharmacokinetics, and effect on the insulin-like growth factor axis," Cancer Research, vol. 70, no. 22, pp. 9003-9011, 2010.

[33] R. M. R. Gangemi, F. Griffero, D. Marubbi et al., "SOX2 silencing in glioblastoma tumor-initiating cells causes stop of proliferation and loss of tumorigenicity," Stem Cells, vol. 27, no. 1, pp. 40-48, 2009.

[34] J. Ryu, B. M. Ku, Y. K. Lee et al., "Resveratrol reduces tnf-alphainduced u373mg human glioma cell invasion through regulating nf-kappab activation and upa/upar expression," Anticancer Research, vol. 31, pp. 4223-4230, 2011.

[35] E. C. Filippi-Chiela, M. P. Thomé, M. M. Bueno e Silva et al., "Resveratrol abrogates the Temozolomide-induced G2 arrest leading to mitotic catastrophe and reinforces the Temozolomide-induced senescence in glioma cells," BMC Cancer, vol. 147, pp. 1-13, 2013.

[36] C. Cilibrasi, G. Riva, G. Romano et al., "Resveratrol impairs glioma stem cells proliferation and motility by modulating the wnt signaling pathway," PLoS ONE, vol. 12, no. 1, Article ID e0169854, 2017.
[37] I. Paul, S. Bhattacharya, A. Chatterjee, and M. K. Ghosh, "Current Understanding on EGFR and Wnt/ $\beta$-Catenin Signaling in Glioma and Their Possible Crosstalk," Genes \& Cancer, vol. 4, no. 11-12, pp. 427-446, 2013.

[38] S. Gotze, M. Wolter, G. Reifenberger, O. Müller, and S. Sievers, "Frequent promoter hypermethylation of Wnt pathway inhibitor genes in malignant astrocytic gliomas," International Journal of Cancer, vol. 126, no. 11, pp. 2584-2593, 2010.

[39] K. H. Kim, H. J. Seol, E. H. Kim et al., "Wnt/ $\beta$-catenin signaling is a key downstream mediator of MET signaling in glioblastoma stem cells," Neuro-Oncology, vol. 15, no. 2, pp. 161-171, 2013.

[40] Y. Zhang, S.-X. Wang, J.-W. Ma et al., "EGCG inhibits properties of glioma stem-like cells and synergizes with temozolomide through downregulation of P-glycoprotein inhibition," Journal of Neuro-Oncology, vol. 121, no. 1, pp. 41-52, 2015.

[41] T. C. Chen, W. Wang, E. B. Golden et al., "Green tea epigallocatechin gallate enhances therapeutic efficacy of temozolomide in orthotopic mouse glioblastoma models," Cancer Letters, vol. 302, no. 2, pp. 100-108, 2011.

[42] M. Nihal, H. Ahsan, I. A. Siddiqui, H. Mukhtar, N. Ahmad, and G. S. Wood, “(-)-Epigallocatechin-3-gallate (EGCG) sensitizes melanoma cells to interferon induced growth inhibition in a mouse model of human melanoma," Cell Cycle, vol. 8, no. 13, pp. 2057-2063, 2009.

[43] H. Chen, C. N. Landen, Y. Li, R. D. Alvarez, and T. O. Tollefsbol, "Epigallocatechin gallate and sulforaphane combination treatment induce apoptosis in paclitaxel-resistant ovarian cancer cells through hTERT and Bcl-2 down-regulation," Experimental Cell Research, vol. 319, no. 5, pp. 697-706, 2013.

[44] H. K. Lee, C. Xiang, S. Cazacu et al., "GRP78 is overexpressed in glioblastomas and regulates glioma cell growth and apoptosis," Neuro-Oncology, vol. 10, no. 3, pp. 236-243, 2008.

[45] B. N. Singh, S. Shankar, and R. K. Srivastava, "Green tea catechin, epigallocatechin-3-gallate (EGCG): mechanisms, perspectives and clinical applications," Biochemical Pharmacology, vol. 82, no. 12, pp. 1807-1821, 2011.

[46] G. Liang, A. Tang, X. Lin et al., "Green tea catechins augment the antitumor activity of doxorubicin in an in vivo mouse model for chemoresistant liver cancer," International Journal of Oncology, vol. 37, no. 1, pp. 111-123, 2010.

[47] C. Li, C. Zhou, S. Wang et al., "Sensitization of glioma cells to tamoxifen-induced apoptosis by pl3-kinase inhibitor through the gsk-3 $\beta / \beta$-catenin signaling pathway," PLoS ONE, vol. 6, no. 10, Article ID e27053, 2011.

[48] S. Bettuzzi, M. Brausi, F. Rizzi, G. Castagnetti, G. Peracchia, and A. Corti, "Chemoprevention of human prostate cancer by oral administration of green tea catechins in volunteers with highgrade prostate intraepithelial neoplasia: a preliminary report from a one-year proof-of-principle study," Cancer Research, vol. 66, no. 2, pp. 1234-1240, 2006.

[49] A. Jatoi, N. Ellison, P. A. Burch et al., "A phase II trial of green tea in the treatment of patients with androgen independent metastatic prostate carcinoma," Cancer, vol. 97, no. 6, pp. 14421446, 2003.

[50] T. D. Shanafelt, Y. K. Lee, T. G. Call et al., "Clinical effects of oral green tea extracts in four patients with low grade B-cell malignancies," Leukemia Research, vol. 30, no. 6, pp. 707-712, 2006.

[51] F. Manero, F. Gautier, T. Gallenne et al., "The small organic compound HA14-1 prevents Bcl-2 interaction with bax to sensitize malignant glioma cells to induction of cell death," Cancer Research, vol. 66, no. 5, pp. 2757-2764, 2006. 
[52] C. Liu, Y. Tu, X. Sun et al., "Wnt/beta-Catenin pathway in human glioma: expression pattern and clinical/prognostic correlations," Clinical and Experimental Medicine, vol. 11, no. 2, pp. 105-112, 2013.

[53] A. R. Mawson, "Retinoids in the treatment of glioma: A new perspective," Cancer Management and Research, vol. 4, no. 1, pp. 233-241, 2012.

[54] C. P. Haar, P. Hebbar, G. C. Wallace IV et al., "Drug resistance in glioblastoma: A mini review," Neurochemical Research, vol. 37, no. 6, pp. 1192-1200, 2012.

[55] S. M. Lippman and R. Lotan, "Advances in the development of retinoids as chemopreventive agents," Journal of Nutrition, vol. 130, pp. 479S-482S, 2000.

[56] A. Das, N. L. Banik, and S. K. Ray, "N-(4-Hydroxyphenyl) retinamide induced both differentiation and apoptosis in human glioblastoma T98G and U87MG cells," Brain Research, vol. 1227, pp. 207-215, 2008.

[57] M. Mellai, V. Caldera, A. Patrucco, L. Annovazzi, and D. Schiffer, "Survivin expression in glioblastomas correlates with proliferation, but not with apoptosis," Anticancer Reseach, vol. 28, no. 1 A, pp. 109-118, 2008.

[58] J. George, N. L. Banik, and S. K. Ray, "Survivin knockdown and concurrent 4-HPR treatment controlled human glioblastoma in vitro and in vivo," Neuro-Oncology, vol. 12, no. 11, pp. 1088-1101, 2010.

[59] S. D. McAllister, C. Chan, R. J. Taft et al., "Cannabinoids selectively inhibit proliferation and induce death of cultured human glioblastoma multiforme cells," Journal of Neuro-Oncology, vol. 74, pp. 31-40, 2005.

[60] S. D. McAllister, L. Soroceanu, and P.-Y. Desprez, "The Antitumor Activity of Plant-Derived Non-Psychoactive Cannabinoids," Journal of Neuroimmune Pharmacology, vol. 10, no. 2, pp. 255-267, 2015.

[61] D. I. Abrams, "Integrating cannabis into clinical cancer care," Current Oncology, vol. 23, no. 2, pp. S8-S14, 2016.

[62] J. P. Marcu, R. T. Christian, D. Lau et al., "Cannabidiol enhances the inhibitory effects of $\Delta 9$ - tetrahydrocannabinol on human glioblastoma cell proliferation and survival," Molecular Cancer Therapeutics, vol. 9, no. 1, pp. 180-189, 2010.

[63] G. Galanti, T. Fisher, I. Kventsel et al., " $\Delta 9$-Tetrahydrocannabinol inhibits cell cycle progression by downregulation of E2F1 in human glioblastoma multiforme cells," Acta Oncologica, vol. 47, no. 6, pp. 1062-1070, 2008.

[64] S. Torres, M. Lorente, F. Rodríguez-Fornés et al., "A combined preclinical therapy of cannabinoids and temozolomide against glioma," Molecular Cancer Therapeutics, vol. 10, no. 1, pp. 90103, 2011.

[65] M. Guzman, M. J. Duarte, C. Blázquez et al., "A pilot clinical study of $\Delta 9$-tetrahydrocannabinol in patients with recurrent glioblastoma multiforme," British Journal of Cancer, vol. 95, no. 2, pp. 197-203, 2006.

[66] B. Valle-Argos, D. Gómez-Nicola, and M. Nieto-Sampedro, "Glioma growth inhibition by neurostatin and O-But GD1b," Neuro-Oncology, vol. 12, no. 11, pp. 1135-1146, 2010.

[67] B. Valle-Argos, D. Gómez-Nicola, and M. Nieto-Sampedro, "Neurostatin blocks glioma cell cycle progression by inhibiting EGFR activation," Molecular and Cellular Neuroscience, vol. 46, no. 1, pp. 89-100, 2011.

[68] J. Abad-Rodriguez, M. Bernabé, L. Romero-Ramírez, M. Vallejo-Cremades, A. Fernández-Mayoralas, and M. NietoSampedro, "Purification and structure of neurostatin, an inhibitor of astrocyte division of mammalian brain," Journal of Neurochemistry, vol. 74, no. 6, pp. 2547-2556, 2000.

[69] E. Doncel-Perez, I. Garcia-Alvarez, and A. Fernandez-Mayoralas, "Synthetic glycolipids for glioma growth inhibition developed from neurostatin and NF115 compound," Bioorganic Medicinal Chemistry Letters, vol. 15, no. 2, pp. 435-439, 2013.

[70] M. Nieto-Sampedro and V. C. Muneton-Gomez, "Neuronal glycolipids regulate glial cell division negatively during development and following a lesion," Revista de Neurologia, vol. 16, no. 64, pp. 549-567, 2017.

[71] W. Tian, Z. Deng, and K. Hong, "The Biological Activities of Sesterterpenoid-Type Ophiobolins," Marine Drug, vol. 15, no. 7, p. 229, 2017.

[72] M. Bury, E. Novo-Uzal, A. Andolfi et al., "Ophiobolin A, a sesterterpenoid fungal phytotoxin, displays higher in vitro growth-inhibitory effects in mammalian than in plant cells and displays in vivo antitumor activity," International Journal of Oncology, vol. 43, no. 2, pp. 575-585, 2013.

[73] M. Bury, A. Girault, V. Megalizzi et al., "Ophiobolin A induces paraptosis-like cell death in human glioblastoma cells by decreasing BKCa channel activity," Cell Death \& Disease, vol. 4, no. 3, p. e561, 2013.

[74] R. Morrison, T. Lodge, A. Evidente, R. Kiss, and H. Townley, "Ophiobolin A, a sesterpenoid fungal phytotoxin, displays different mechanisms of cell death in mammalian cells depending upon the cancer cell origin," International Journal of Oncology, vol. 50, no. 3, pp. 773-786, 2017.

[75] A. Bregy, A. H. Shah, M. V. Diaz et al., "The role of Gliadel wafers in the treatment of high-grade gliomas," Expert Review of Anticancer Therapy, vol. 13, no. 12, pp. 1453-1461, 2013.

[76] M. Panigrahi, P. K. Das, and P. M. Parikh, "Brain tumor and Gliadel wafer treatment," Indian Journal of Cancer, vol. 48, no. 1, pp. 11-17, 2011.

[77] P. Miglierini, M. Bouchekoua, B. Rousseau, P. Dam Hieu, J.-P. Malhaire, and O. Pradier, "Impact of the per-operatory application of GLIADEL wafers (BCNU, carmustine) in combination with temozolomide and radiotherapy in patients with glioblastoma multiforme: efficacy and toxicity," Clinical Neurology and Neurosurgery, vol. 114, no. 9, pp. 1222-1225, 2012.

[78] M. Westphal, D. C. Hilt, E. Bortey et al., "A phase 3 trial of local chemotherapy with biodegradable carmustine (BCNU) wafers (Gliadel wafers) in patients with primary malignant glioma," Neuro-Oncology, vol. 5, no. 2, pp. 79-88, 2003.

[79] H. Brem, S. Piantadosi, P. C. Burger et al., "Placebo-controlled trial of safety and efficacy of intraoperative controlled delivery by biodegradable polymers of chemotherapy for recurrent gliomas," The Lancet, vol. 345, no. 8956, pp. 1008-1012, 1995.

[80] M. J. McGirt, K. D. Than, J. D. Weingart et al., "Gliadel (BCNU) wafer plus concomitant temozolomide therapy after primary resection of glioblastoma multiforme: Clinical article," Journal of Neurosurgery, vol. 110, no. 3, pp. 583-588, 2009.

[81] G. L. Gallia, S. Brem, and H. Brem, "Local treatment of malignant brain tumors using implantable chemotherapeutic polymers," JNCCN - Journal of the National Comprehensive Cancer Network, vol. 3, no. 5, pp. 721-728, 2005.

[82] E. L. Weber and E. A. Goebel, "Cerebral edema associated with Gliadel wafers: Two case studies," Neuro-Oncology, vol. 7, no. 1, pp. 84-89, 2005.

[83] J. Kreuter, "Influence of the surface properties on nanoparticlemediated transport of drugs to the brain," Journal of Nanoscience and Nanotechnology, vol. 4, no. 5, pp. 484-488, 2004. 
[84] D. Peer, J. M. Karp, S. Hong, O. C. Farokhzad, R. Margalit, and R. Langer, "Nanocarriers as an emerging platform for cancer therapy," Nature Nanotechnology, vol. 2, no. 12, pp. 751-760, 2007.

[85] D. J. Begley and M. W. Brightman, "Structural and functional aspects of the blood-brain barrier," Progress in Drug Research, vol. 61, pp. 39-78, 2003.

[86] J. F. Deeken and W. Löscher, "The blood-brain barrier and cancer: Transporters, treatment, and trojan horses," Clinical Cancer Research, vol. 13, no. 6, pp. 1663-1674, 2007.

[87] W. M. Pardridge, "Blood-brain barrier drug targeting: the future of brain drug development," Molecular Interventions, vol. 3, no. 2, pp. 90-51, 2003.

[88] R. D. Egleton and T. P. Davis, "Development of neuropeptide drugs that cross the blood-brain barrier," Neurotherapeutics, vol. 2, no. 1, pp. 44-53, 2005.

[89] W. M. Pardridge, "Molecular Trojan horses for blood-brain barrier drug delivery," Current Opinion in Pharmacology, vol. 6, no. 5, pp. 494-500, 2006.

[90] G. F. Woodworth, G. P. Dunn, E. A. Nance, J. Hanes, and H. Brem, "Emerging insights into barriers to effective brain tumor therapeutics," Frontiers in Oncology, vol. 4, no. 126, pp. 1-14, 2014.

[91] S.-S. Kim, A. Rait, F. Rubab et al., "The clinical potential of targeted nanomedicine: Delivering to cancer stem-like cells," Molecular Therapy, vol. 22, no. 2, pp. 278-291, 2014.

[92] J. Panyam and V. Labhasetwar, "Sustained cytoplasmic delivery of drugs with intracellular receptors using biodegradable nanoparticles," Molecular Pharmacology, vol. 1, no. 1, pp. 77-84, 2004.

[93] E. Allard, C. Passirani, and J.-P. Benoit, "Convection-enhanced delivery of nanocarriers for the treatment of brain tumors," Biomaterials, vol. 30, no. 12, pp. 2302-2318, 2009.

[94] Y. Li, H. He, X. Jia, W.-L. Lu, J. Lou, and Y. Wei, "A dualtargeting nanocarrier based on poly(amidoamine) dendrimers conjugated with transferrin and tamoxifen for treating brain gliomas," Biomaterials, vol. 33, no. 15, pp. 3899-3908, 2012.

[95] B. K. Hendricks, A. A. Cohen-Gadol, and J. C. Miller, "Novel delivery methods bypassing the blood-brain and blood-tumor barriers," Neurosurgical Focus, vol. 38, no. 3, p. E10, 2015.

[96] J. C. K. Lai, G. Ananthakrishnan, S. Jandhyam et al., “Treatment of human astrocytoma U87 cells with silicon dioxide nanoparticles lowers their survival and alters their expression of mitochondrial and cell signaling proteins," International Journal of Nanomedicine, vol. 5, no. 1, pp. 715-723, 2010.

[97] L. Bobyk, M. Edouard, P. Deman et al., "Photoactivation of gold nanoparticles for glioma treatment," Nanomedicine: Nanotechnology, Biology and Medicine, vol. 9, no. 7, pp. 10891097, 2013.

[98] M. Aryal, J. Park, N. Vykhodtseva, Y.-Z. Zhang, and N. McDannold, "Enhancement in blood-tumor barrier permeability and delivery of liposomal doxorubicin using focused ultrasound and microbubbles: Evaluation during tumor progression in a rat glioma model," Physics in Medicine and Biology, vol. 60, no. 6, pp. 2511-2527, 2015.

[99] K. Sang-Soo, J. B. Harford, K. F. Pirollo et al., "Effective treatment of glioblastoma requires crossing the bloodbrain barrier and targeting tumors including cancer stem cells: the promise of nanomedicine," Biochemical and Biophysical Research Communications, vol. 468, no. 3, pp. 485-489, 2015.
[100] L. Juillerat-Jeanneret, "The targeted delivery of cancer drugs across the blood-brain barrier: chemical modifications of drugs or drug-nanoparticles?" Drug Discovery Therapy, vol. 13, no. 2324, pp. 1099-1106, 2008. 


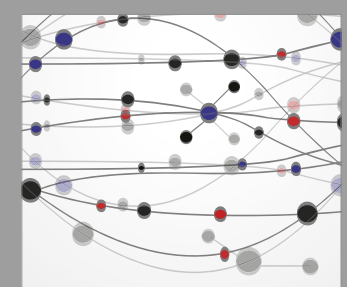

The Scientific World Journal
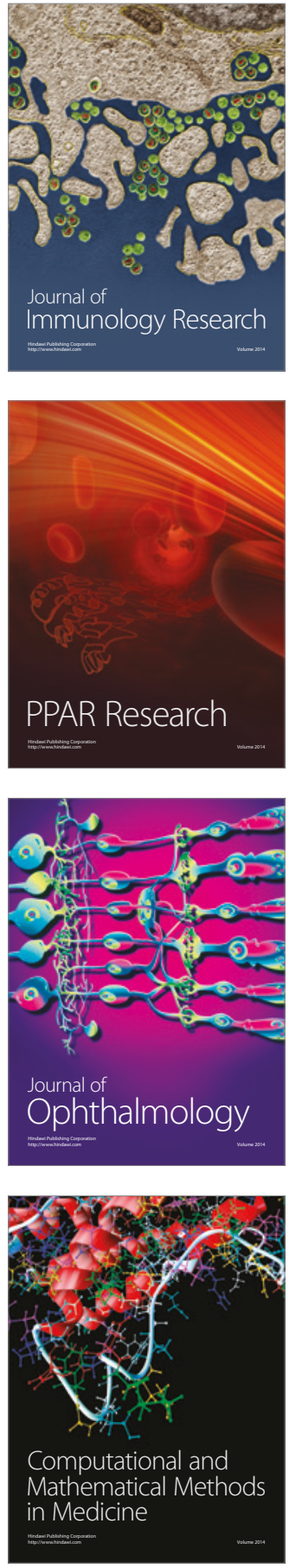

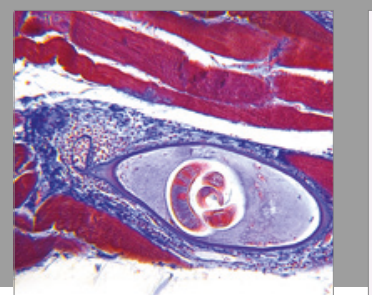

Gastroenterology Research and Practice
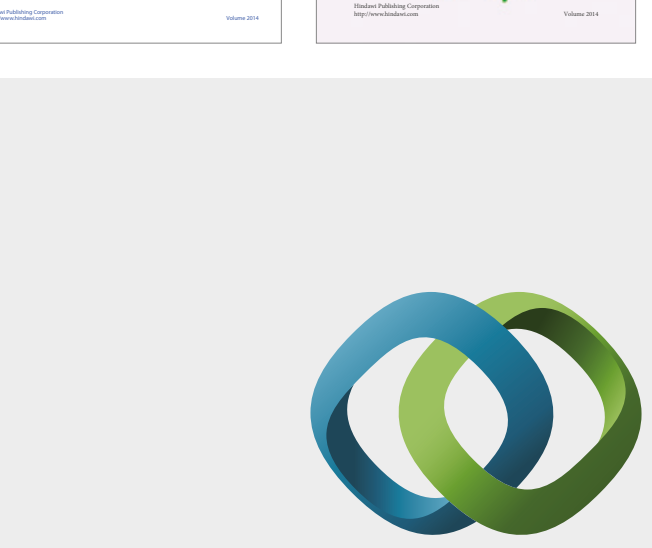

\section{Hindawi}

Submit your manuscripts at

https://www.hindawi.com
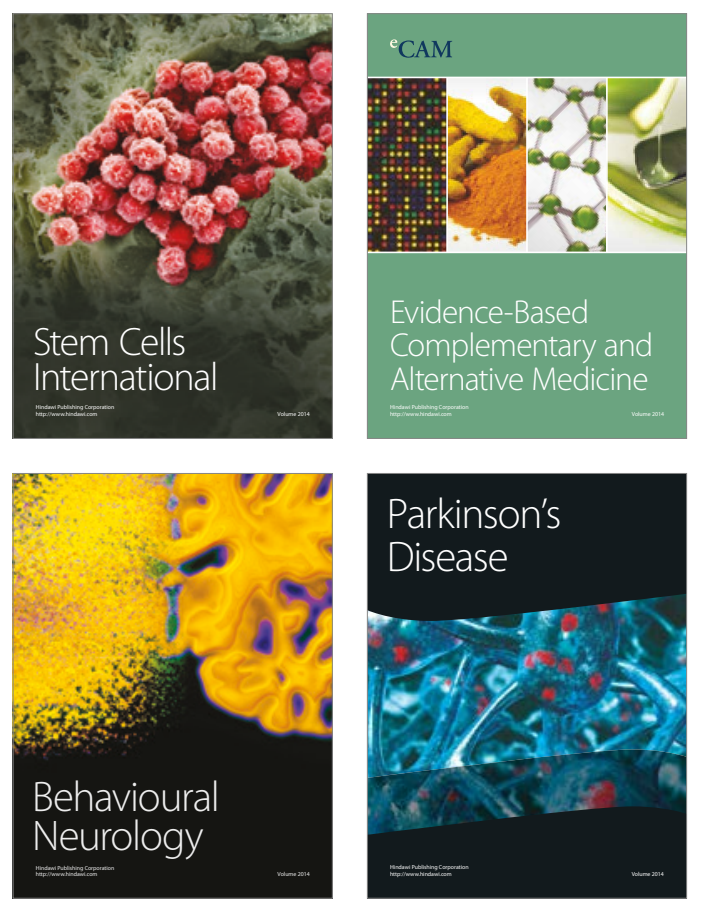
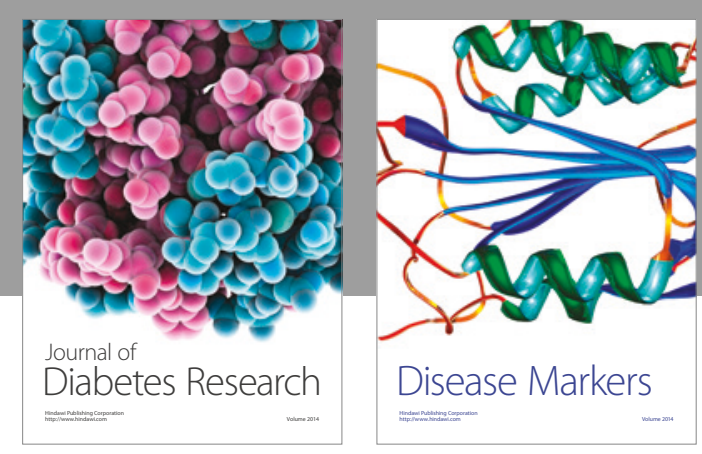

Disease Markers
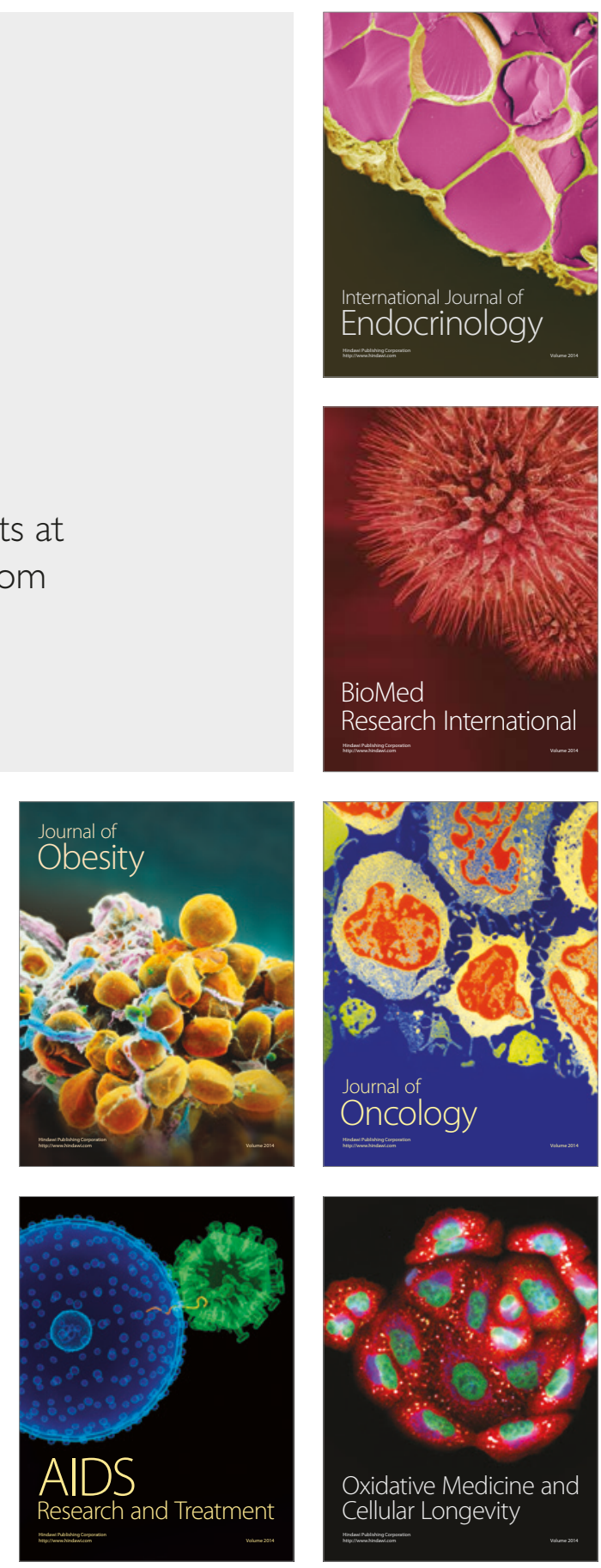